\title{
Compressive Neuropathy of the Posterior Tibial Nerve at the Lower Calf Caused by a Ruptured Intramuscular Baker Cyst
}

\author{
Seock Ho Moon, MD, Sun Im, MD, Geun-Young Park, MD, Su-Jin Moon, MD, \\ Hye-Jeong Park, MD, Hyun-Sook Choi, MD, Yil Ryun Jo, MD \\ Department of Rehabilitation Medicine, Bucheon St. Mary's Hospital, \\ The Catholic University of Korea College of Medicine, Bucheon, Korea
}

Baker cyst is an enlargement of the gastrocnemius-semimembranosus bursa. Neuropathy can occur due to either direct compression from the cyst itself or indirectly after cyst rupture. We report a unique case of a 49-year-old man with left sole pain and paresthesia who was diagnosed with posterior tibial neuropathy at the lower calf area, which was found to be caused by a ruptured Baker cyst. The patient's symptoms resembled those of lumbosacral radiculopathy and tarsal tunnel syndrome. Posterior tibial neuropathy from direct pressure of ruptured Baker cyst at the calf level has not been previously reported. Ruptured Baker cyst with resultant compression of the posterior tibial nerve at the lower leg should be included in the differential diagnosis of patients who complain of calf and sole pain. Electrodiagnostic examination and imaging studies such as ultrasonography or magnetic resonance imaging should be considered in the differential diagnosis of isolated paresthesia of the lower leg.

Keywords Tibial neuropathy, Nerve compression syndromes, Popliteal cyst

\section{INTRODUCTION}

Baker cyst develops from enlargement of the gastrocnemius-semimembranosus bursa [1,2]. Although most Baker cysts commonly present as an asymptomatic mass

Received July 10, 2012; Accepted September 17, 2012

Corresponding author: Yil Ryun Jo

Department of Rehabilitation Medicine, The Catholic University of Korea College of Medicine, 327 Sosa-ro, Wonmi-gu, Bucheon 420-717, Korea Tel: +82-32-340-2170, Fax: +82-32-340-2173, E-mail: xpops83@hanmail. net

(c) This is an open-access article distributed under the terms of the Creative Commons Attribution Non-Commercial License (http://creativecommons. org/licenses/by-nc/3.0) which permits unrestricted noncommercial use, distribution, and reproduction in any medium, provided the original work is properly cited.

Copyright $\odot 2013$ by Korean Academy of Rehabilitation Medicine occurring just below the popliteal fossa, they may cause clinical problems such as thrombophlebitis, compartment syndrome, and entrapment neuropathy [1-4]. Among those, neuropathy can occur either from the cyst itself or after cyst rupture [3]. Rupture or dissection of the cyst causes extravasation of fluid into the calf, which can result in nerve compression caused by posterior compartment syndrome [5]. Whereas compressive neuropathy by intact Baker cyst have been reported $[3,4]$, posterior tibial neuropathy from a ruptured or dissected Baker cyst at the calf level has not been yet reported. We report a case of ruptured Baker cyst as causing posterior compartment syndrome and tibial neuropathy and review the literature on posterior tibial neuropathy by Baker cyst. 


\section{CASE REPORT}

A 49-year-old man with left sole paresthesia and calf pain for 2 weeks was admitted to the department of rheumatology. This pain had a sudden-onset and caused the excruciating calf pain at nights. His medical history was significant for rheumatoid arthritis diagnosed 6 months prior to this and significant also for intermittent low back pain. The patient had bilateral knee joint arthroscopic synovectomy at a local orthopedic clinic 3 months before to manage synovial hyperplasia caused by rheumatoid arthritis. Magnetic resonance imaging (MRI) study of the lumbar spine, at the time of admission, revealed bilateral foraminal spinal stenosis at L3-4 and L4-5 levels. Thus, he was referred to the department of anesthesiology and received diagnostic epidural steroid nerve block at the corresponding levels. However, the symptoms did not improved after the block, and the patient was referred to our physical medicine and rehabilitation department for electrodiagnostic study and further evaluation.

Neurological examination at the time of referral visit showed no significant muscle atrophy but manual muscle test revealed a grade-IV muscle strength in left ankle plantar flexors and a grade-I muscle strength in left great and little toe abductors. Paresthesia was noted at the left sole area. Nerve conduction study showed significantly reduced amplitudes of compound muscle action potential in the left tibial nerve. Mixed nerve studies of the medial and lateral plantar nerves showed no sensory nerve action potential (SNAP) responses. Sural and superficial peroneal nerves showed normal latencies and SNAP amplitudes (Table 1). Denervation potentials were observed at the left abductor hallucis and abductor digiti minimi muscles with discrete recruitment patterns, but no denervation potentials were observed in the gastrocnemius or tibialis posterior muscles (Table 2). On the basis of these results, a preliminary diagnosis of posterior tibial entrapment neuropathy around the tarsal tunnel was made.

To rule out any structural lesion or mass at the tarsal tunnel, a musculoskeletal ultrasonography (US) was performed. However, this failed to identify any discrete mass around the tarsal tunnel area or any echo intensity change of the posterior tibial nerve. Additional proximal ultrasonography scans along the left tibial nerve from the proximal knee did identify a cystic anechoic compressible mass around the left posterior tibial nerve at the mid one-third area of the lower leg (Fig. 1). The cross-sectional area of the left posterior tibial nerve at the location of maximal nerve swelling measured $0.21 \mathrm{~cm}^{2}$. The crosssectional area of the contralateral posterior tibial nerve at the same location measured $0.12 \mathrm{~cm}^{2}$. Subsequently, a MRI study of left lower leg revealed a Baker cyst with intermuscular and intramuscular dissection. The cystic lesion extended from the popliteal fossa, between the two heads of the gastrocnemius muscle to the deep posterior compartment of the middle one-third of the lower leg. Its longitudinal length was about $18.7 \mathrm{~cm}$, and there was no connection with the intra-articular space. Upon gadolinium-infusion, deep flexor muscles near the cyst and at the

Table 1. Findings from nerve conduction studies

\begin{tabular}{|c|c|c|c|c|c|c|c|}
\hline \multirow{2}{*}{ Nerve } & \multirow{2}{*}{ Stimulation site } & \multicolumn{3}{|c|}{ Right } & \multicolumn{3}{|c|}{ Left } \\
\hline & & LAT & AMP & $\mathrm{CV}(\mathrm{m} / \mathrm{s})$ & LAT & AMP & $\mathrm{CV}(\mathrm{m} / \mathrm{s})$ \\
\hline \multicolumn{8}{|l|}{ Motor } \\
\hline \multirow[t]{2}{*}{ Tibial } & Ankle & 4.0 & 10.7 & - & 4.3 & $1.1^{\mathrm{a})}$ & - \\
\hline & Popliteal fossa & 11.3 & 8.6 & 45 & $16.8^{\mathrm{a})}$ & $0.6^{\mathrm{a})}$ & $29^{\text {a) }}$ \\
\hline \multicolumn{8}{|l|}{ Sensory } \\
\hline Superficial peroneal & Lower leg & 3.4 & 13 & - & 3.5 & 14 & - \\
\hline Sural & Lower leg & 3.2 & 11 & - & 3.4 & 10 & - \\
\hline \multicolumn{8}{|l|}{ Mixed nerve } \\
\hline Medial plantar & Medial sole & 2.6 & 5 & - & $\mathrm{NE}$ & NE & - \\
\hline Lateral plantar & Lateral sole & 3.1 & 3 & - & NE & NE & - \\
\hline
\end{tabular}

LAT, latency (motor, onset latency; sensory and mixed nerve, peak latency); AMP, amplitude (motor, mV; sensory and mixed nerve, $\mu \mathrm{V}) ; \mathrm{CV}$, conduction velocity; NE, not evoked.

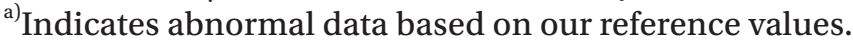


periphery of the cyst were enhanced indicating rupture of the Baker cyst. Therefore, we concluded that posterior tibial neuropathy was caused by a direct compression from the ruptured intramuscular Baker cyst (Fig. 2).

Careful re-examination of the leg muscle function

Table 2. Needle electromyographic findings

\begin{tabular}{|c|c|c|c|c|}
\hline \multirow{2}{*}{$\begin{array}{l}\text { Muscle } \\
\text { (left side) }\end{array}$} & \multicolumn{2}{|c|}{ ASA } & \multirow{2}{*}{ MUAP } & \multirow{2}{*}{$\begin{array}{c}\text { Inter- } \\
\text { ference } \\
\text { pattern }\end{array}$} \\
\hline & PSW & Fib & & \\
\hline $\begin{array}{l}\text { Gastrocnemius, } \\
\text { medial }\end{array}$ & None & None & Normal & Reduced \\
\hline Soleus & None & None & Normal & Reduced \\
\hline Tibialis posterior & None & None & Normal & Reduced \\
\hline $\begin{array}{l}\text { Flexor digitorum } \\
\text { longus }\end{array}$ & + & + & Normal & Discrete \\
\hline $\begin{array}{l}\text { Flexor hallucis lon- } \\
\text { gus }\end{array}$ & + & None & Normal & Discrete \\
\hline Abductor hallucis & + & None & Normal & Discrete \\
\hline $\begin{array}{l}\text { Abductor digit } \\
\text { minimi }\end{array}$ & + & None & Normal & Discrete \\
\hline Gluteus medius & None & None & Normal & Full \\
\hline Gluteus maximus & None & None & Normal & Full \\
\hline Vastus medialis & None & None & Normal & Full \\
\hline Tibialis anterior & None & None & Normal & Full \\
\hline $\begin{array}{l}\text { Extensor hallucis } \\
\text { longus }\end{array}$ & None & None & Normal & Full \\
\hline Paraspinalis, L2-S1 & None & None & & \\
\hline
\end{tabular}

ASA, abnormal spontaneous activity; PSW, positive sharp wave; Fib, fibrillation potential; MUAP, motor unit action potential. showed mild weakness of the left great toe and digit flexor muscles. Additional needle electromyography study was performed, which showed denervation potentials in the left flexor hallucis longus and flexor digitorum longus muscles; however, no denervation potentials were observed in the left tibialis posterior, gastrocnemius, or soleus muscles. He was ultimately diagnosed with a posterior tibial entrapment neuropathy between the tarsal tunnel and popliteal space, distal to the tibialis posterior muscle branching site, secondary to a ruptured Baker cyst.

The patient was treated with a regiment consisting of gabapentin Neurontin cap 1,200 mg (Pfizer Korea Ltd., Seoul, Korea), transcutaneous electrical nerve stimulation, electrical stimulation therapy, and physical therapy. Additionally, a referral was made to the department of orthopedic surgery for possible surgical intervention. However, the patient's symptoms improved substantially after non-invasive treatment. There being no evidence of infection or other complications, and because the small cyst was located between the deep flexor muscles and blood vessels, the risk of an aspiration was deemed too great. Therefore, the department of orthopedic surgery and the patient decided to continue the non-operative course of management, and after two months of medical and physical therapy, the patient expressed significant improvement of symptoms.

\section{DISCUSSION}

Enlargement of the gastrocnemius-semimembranosus bursa is known as a Baker cyst $[1,3,6]$. The cyst can be
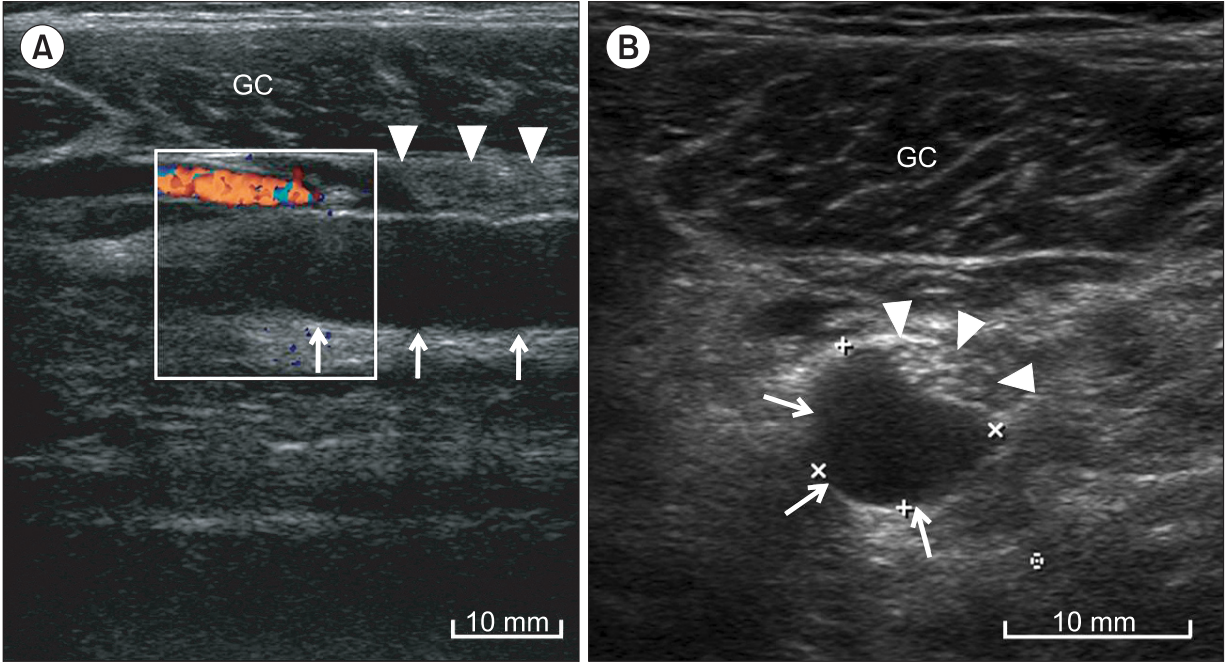

Fig. 1. (A) Longitudinal and (B) transverse ultrasonographic imaging of the lower leg showed hypoechoic cyst (arrows) along the tibial nerve (arrow heads). GC, gastrocnemius. 

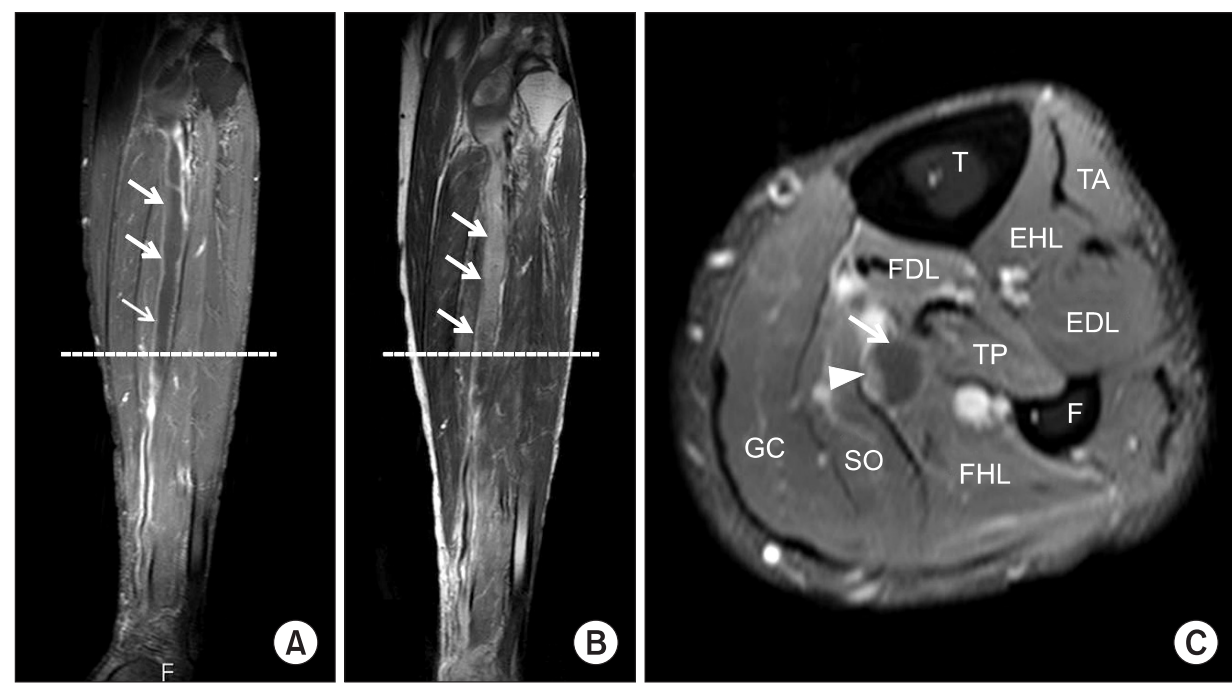

Fig. 2. (A) T1-weighted contrast enhanced coronal and (B) T2-weighted coronal magnetic resonance imaging (MRI) of the lower leg showed an elongated cystic lesion (arrows) with peripheral enhancement adjacent to the tibial nerve. (C) T1-weigted contrast enhanced transverse MRI of the lower leg showed tibial nerve (arrow head) entrapped by the cyst (arrow). T, tibia; F, fibula; TA, tibialis anterior; EHL, extensor hallucis longus; EDL, extensor digitorum longus; FDL, flexor digitorum longus; TP, tibialis posterior; FHL, flexor hallucis longus; SO, soleus; GC, gastrocnemius.

formed from an inter-communication between the knee joint and the bursa, and in such cases, it is often associated with an intra-articular pathology of the knee, such as osteoarthritis or rheumatoid arthritis [7]. Non-communicating cysts, however, often have no associated knee pathology and are thought to be primary enlargements of the gastrocnemius-semimembranosus bursa $[1,3]$.

Although the chief complaint in most cases of Baker cysts is an asymptomatic mass, it is common for patients to experience swelling, pain, or stiffness of the knee often aggravated by walking. Other symptoms include bulging and tightness in the popliteal area [1].

Baker cysts may rupture and subsequently induce calf symptoms or signs, which can similarly be caused by large, intact popliteal cysts without calf extension [1]. Since most cysts originate from the gastrocnemiussemimembranosus bursa, they are located in the mid to superficial popliteal tissues [3] and may compress an adjacent neurovascular bundle [2]. Ruptured Baker cysts can cause lower leg and ankle swelling with pain that is clinically indistinguishable from that associated with acute deep vein thrombosis. For this reason, ruptured Baker cysts have also been called pseudothrombophlebitis $[1,2,6]$. Occasionally, the ruptured of these cysts may cause tibial nerve entrapment and demonstrate sensory symptoms such as paresthesia, numbness, or pain, and occasionally, they are known to gastrocnemius muscle atrophy [6].

For accuracy of diagnosis, electromyography with nerve conduction study is commonly recommended for patients who exhibit neurologic symptoms. The relatively inexpensive and noninvasive ultrasonography of popliteal cysts generally would be the first choice because it can detect lesions in calf that might also extended to thigh and can image the veins to reveal thrombosis. Further imaging studies such as an MRI or computed tomography can be considered as necessary $[1,6]$.

Nerve compression is an unusual complication from Baker cyst, and to date, only few such cases have been reported [4]. In these reports, early decompression by joint aspiration, synovectomy, or open resection had resulted in clinical improvement of the neurologic symptoms [4]. In the case presented here, conservative treatment with oral medication and physical modalities was successful in alleviating the patient's symptoms. However, further future clinical studies, including electrodiagnostic and follow-up imaging studies, would be needed to fully elucidate the long-term prognosis of tibial neuropathy caused by intramuscular Baker cyst.

In summary, we have presented an unusual case of peripheral compressive neuropathy caused by a ruptured Baker cyst. The patient exhibited symptoms which resembled those of lumbosacral radiculopathy and tarsal tunnel syndrome. Among the patients who complain of 
calf and sole pain, compression neuropathy of the posterior tibial leg should be included in the differential diagnosis. Along with a careful neurologic examination, electrodiagnostic studies, and complimentary imaging studies, such as musculoskeletal ultrasonography or MRI, should be performed in the differential diagnosis of sole paresthesia of lower leg.

\section{CONFLICT OF INTEREST}

No potential conflict of interest relevant to this article was reported.

\section{REFERENCES}

1. Handy JR. Popliteal cysts in adults: a review. Semin Arthritis Rheum 2001;31:108-18.

2. Sanchez JE, Conkling N, Labropoulos N. Compression syndromes of the popliteal neurovascular bundle due to Baker cyst. J Vasc Surg 2011;54:1821-9.

3. Dash S, Bheemreddy SR, Tiku ML. Posterior tibial neuropathy from ruptured Baker's cyst. Semin Arthritis Rheum 1998;27:272-6.

4. Ji JH, Shafi M, Kim WY, Park SH, Cheon JO. Compressive neuropathy of the tibial nerve and peroneal nerve by a Baker's cyst: case report. Knee 2007;14:249-52.

5. Scott WN, Jacobs B, Lockshin MD. Posterior compartment syndrome resulting from a dissecting popliteal cyst: case report. Clin Orthop Relat Res 1977;(122):189-92.

6. Sato O, Kondoh K, Iyori K, Kimura H. Midcalf ultrasonography for the diagnosis of ruptured Baker's cysts. Surg Today 2001;31:410-3.

7. Liao ST, Chiou CS, Chang CC. Pathology associated to the Baker's cysts: a musculoskeletal ultrasound study. Clin Rheumatol 2010;29:1043-7. 